Noor Dina Hashim, MD

Mohd Razif Mohamad Yunus, MBBS, MS (ORL-HNS)

Marina Mat Baki, MD, MS (ORL-HNS)

Mazita Ami, MBChB, MS (ORL-HNS)

Department of Otorhinolaryngology Universiti Kebangsaan Malaysia Medical Centre

Kuala Lumpur, Malaysia
Correspondence: Mohd Razif Mohamad Yunus, MBBS, MS (ORL-HNS) Department of Otorhinolaryngology

Universiti Kebangsaan Malaysia Medical Centre

Jalan Yaacob Latiff, Bandar Tun Razak 56000

Cheras, Kuala Lumpur, Malaysia

Phone: +60391455555 ext 6845

Fax: +60391737840

Email : razif72@gmail.com

Reprints will not be available from the authors.

The authors declared that this represents original materia that is not being considered for publication or has not been published or accepted for publication elsewhere, in full or in part, in print or electronic media; that the manuscript has been read and approved by all the authors, that the requirements for authorship have been met by each author, and that each author believes that the manuscript represents honest work.

Disclosures: The authors signed disclosures that there are no financial or other (including personal) relationships, intellectual passion, political or religious beliefs, and institutional affiliations that might lead to a conflict of interest.

\title{
Laryngeal Trauma with Phrenic Nerve Injury: A Rare Association
}

\begin{abstract}
Objectives: To share our experience in managing a rare involvement of phrenic nerve injury in laryngeal trauma.
\end{abstract}

\section{Methods:}

\author{
Design: Case Report \\ Setting: Tertiary Referral Centre \\ Patient: One
}

Results: A 23-year-old male sustained blunt laryngeal trauma associated with phrenic nerve injury leading to silent traumatic diaphragmatic paralysis. He underwent tracheotomy and surgical repair of Schaeffer class IV laryngeal injuries, and conservative therapy for the diaphragmatic paralysis, which eventually resolved.

Conclusion: Patients with laryngeal trauma may have concomitant phrenic nerve injury causing diaphragmatic paralysis. The diagnosis should be considered particularly if the patient has respiratory problems despite securing the airway by tracheotomy. A high index of suspicion is required in diagnosing such an association. Patients should be closely monitored even though most will recover as some may present with later morbidities. A search of PubMed and OvidSP using the terms "larynx," "laryngeal trauma" and "phrenic nerve" did not yield any report of phrenic nerve injury in association with laryngeal trauma. To our knowledge, this is may be the first reported case of phrenic nerve injury in association with blunt laryngeal trauma.

Keywords: Iarynx, trauma, phrenic nerve

The incidence of laryngeal trauma in association with phrenic nerve injury is rarely reported in the literature. Phrenic nerve injury may lead to diaphragmatic paralysis. It is usually overlooked as associated laryngeal injuries commonly cause more alarming respiratory difficulties. The pathophysiology of nerve injury may include stretching, transection or compression. We report a case of phrenic nerve injury seen in laryngeal trauma. Methods of diagnosing diaphragmatic paralysis secondary to phrenic nerve injury are also briefly discussed.

\section{CASE REPORT}

A 23-year-old male was involved in a motor vehicle accident when he was riding a motorbike, travelling at medium velocity behind a bus which suddenly stopped. The rear engine hood opened unexpectedly and hit the patient's anterior neck. He had no loss of consciousness but complained of throat pain associated with hoarseness. There was no associated neck pain or paraesthesia or weakness of limbs. On examination, the patient was fully conscious. He was not 


\section{CASE REPORTS}

in respiratory distress. His voice was hoarse, and his neck appeared swollen with subcutaneous emphysema on the right side. There was no open wound but anterior neck bruises were apparent at the level of the hyoid bone which was tender on palpation. Laryngeal crepitus was absent with loss of thyroid prominence. Laryngeal injury was suspected. The trachea was midline. Lung examination showed reduced air entry to the right lower lobe. Other physical examinations were otherwise normal. During the primary survey, the patient was self-ventilating with no signs of distress.

An emergency tracheotomy was performed for airway protection. After the patient was stabilized and the severity of injury identified clinically, he was put under general anaesthesia with ventilation via tracheotomy tube, and direct laryngoscopy and neck exploration was performed. The aryepiglottic folds were oedematous with haematoma collection and mucosal lacerations over the right ventricle and right aryepiglottic fold exposing the right arytenoid cartilage. There were comminuted fractures of the thyroid cartilage. Vocal cord mobility could not be determined due to distorted anatomy at the supraglottic level. There also was a small haematoma on the left strap muscles. The findings were consistent with laryngeal injury Schaeffer-Fuhrman class IV according to the laryngotracheal injury classification proposed by Trone and Schaefer' and modified by Fuhrman et al. ${ }^{2}$ The fractured thyroid cartilages were repaired with prolene 4/0 (Ethicon Inc, a subsidiary of Johnson \& Johnson) and the mucosal lacerations sutured with vicryl $6 / 0$ (Ethicon Inc, a subsidiary of Johnson \& Johnson) to cover exposed cartilage. He was also started on intravenous Ciprofloxacin and Metronidazole in view of the exposed cartilage.

A pre-operative chest radiograph (Figure 1) showed an elevated, immobile right hemi-diaphragm, fixed at the level of the seventh right posterior rib. An abdominal ultrasound confirmed the immobility of the right hemi-diaphragm, but there was no evidence of solid organ injury, organ eventration or free fluid in the abdomen. A cervical CT scan showed no fracture of the cervical spine. A diagnosis of laryngeal trauma with right hemi-diaphragmatic paralysis secondary to traumatic right phrenic nerve palsy was made. Pre-morbidly, this patient had no significant previous respiratory symptoms and he was an active young man.

The patient was subjected to vigorous chest physiotherapy, and started on naso-gastric tube feeding to allow recovery of the larynx. He also developed hospital-acquired pneumonia which resolved with intravenous antibiotics. The patient was carefully monitored and continued to make an otherwise uneventful recovery. A repeat chest $\mathrm{x}$ ray one month after the trauma showed a normal diaphragmatic level. Two months after the trauma, decannulation of the tracheotomy tube

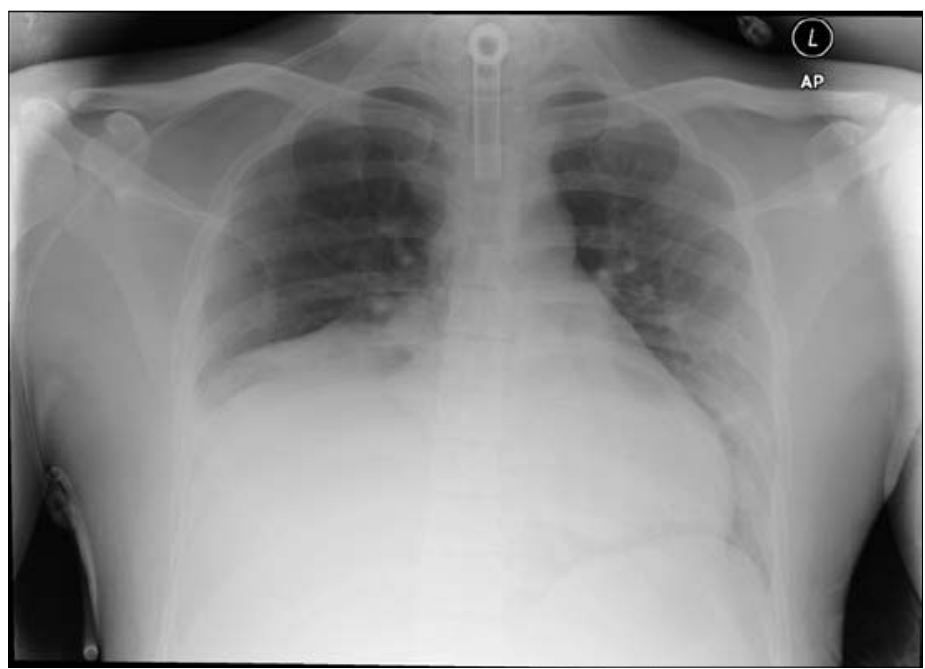

Figure 1. Preoperative chest radiograph showing an elevated right hemi-diaphragm at the level of the seventh right posterior rib.

was done successfully. A swallowing examination showed adequate laryngeal function with minimal pooling, and the tube feeding was gradually discontinued. At six months, indirect laryngoscopy showed excellent healing of laryngeal wounds and normal mobility of bilateral vocal cords. The patient remains asymptomatic after six months.

\section{DISCUSSION}

The phrenic nerve which is formed from $\mathrm{C} 3, \mathrm{C} 4$, and $\mathrm{C} 5$ nerve fibres, descends along the anterior surface of the scalenus anterior muscle of the neck, deep to the sternocleidomastoid muscle, later sandwiched between the subclavian vessels on its way into the thorax. It carries motor and sensory fibres to the diaphragm. Its anatomic location in the neck makes it vulnerable to traumatic injury.

Traumatic phrenic nerve injury is commonly caused by penetrating injury to the neck, blunt trauma to the chest, ${ }^{3}$ jugular or subclavian catheterization, cardiovascular surgery or birth trauma. ${ }^{4}$ Injury may occur at any level along the course of the nerve including neck, chest, heart and abdomen. In trauma cases, hyperextension of neck, cervical injury, chest trauma with rib or lung injury, cardiac trauma such as cardiac tamponade or diaphragmatic rupture may lead to phrenic nerve injury. The pathophysiology of nerve injury may include stretching, transection or compression of the nerve $\mathrm{e}^{5,6}$ as well as chemical injury of the myelin sheaths from cold topical cardioplegia as seen in lung transplants.?

In this particular case, the mechanical force that caused severe laryngeal structure injury may explain involvement of the phrenic nerve. The great impact on the anterior neck may have caused sudden hyperextension. Neck hyperextension could have led to overstretching 
of the phrenic nerve axons without disrupting the integrity of the nerve. The time for recovery would therefore depend on the distance of the nerve injury to the diaphragm, as well as the rate of regeneration. ${ }^{8}$ Although there is also a possibility that phrenic nerve injury may have been due to compression by hematomas of the anterior scalenus or sternocleidomastoid muscles as the nerve runs along these muscles, no severe haematoma of the neck muscles was noted intra-operatively.

Most cases of laryngeal injury present as airway emergencies, obscuring the possibility of respiratory distress contributed by diaphragmatic paralysis following phrenic nerve injury. Most patients with traumatic unilateral diaphragmatic paralysis are usually asymptomatic at the time of the incident. Some will develop dyspnoea with reduced effort tolerance on exertion. A small number of patients may present with respiratory, gastrointestinal or cardiovascular embarrassment as a result of organ eventration, immediately or delayed, months after trauma. ${ }^{6}$ More intense symptoms are observed in patients with bilateral diaphragmatic paralysis.

Clinical examination of the chest in a patient with diaphragmatic paralysis often reveals dullness with reduced movement of the affected chest. Diagnosis is also supported by plain chest radiography which shows an elevated hemi-diaphragm. Lung infection could be a consequence of the paralysis of the hemi-diaphragm whereby the dome lies high in the thorax producing atelectasis and shunting of pulmonary blood flow which can lead to lung infection. ${ }^{9}$

Hemi-diaphragmatic elevation in trauma cases seen in plain radiographs could be caused by phrenic nerve injury causing diaphragmatic paralysis as explained above or may be due to diaphragmatic rupture. Diaphragmatic paralysis may closely mimic diaphragmatic rupture or vice versa. ${ }^{5}$ Valuable information can be obtained from plain chest radiographs. Asymmetry of a hemi-diaphragm is easily seen. The presence of abdominal organs or the placement of a nasogastric tube in an herniated stomach may help in the diagnosis of diaphragmatic rupture. ${ }^{10}$

$\mathrm{CT}$ imaging has increasingly become the mainstay of investigations for suspected diaphragmatic injury. An abrupt discontinuity of the diaphragm and waist like constriction (collar sign) of herniated organs are well seen in cases of diaphragmatic rupture. Sagittal, coronal, and 3-dimensional reformation CT scans may improve sensitivity. ${ }^{10}$ Multiplanar imaging MRI scanning provides a definitive diagnosis of diaphragmatic rupture, and can show intrathoracic herniation of the abdominal viscera. ${ }^{10}$ However, MRI may not readily available in every centre.

Other useful ancillary investigations to confirm or exclude diaphragmatic paralysis or rupture include fluoroscopy and ultrasonographic assessment during respiration. Absent or decreased diaphragmatic motion on these studies is suggestive of diaphragmatic injury. The less popular but valuable barium studies confirm the diagnosis by showing herniated viscera above the diaphragm and constriction through the diaphragmatic tear.

As in our case, the main aim of treatment is to secure the airway. Most laryngeal trauma requires emergency tracheotomy in the setting of an edematous and severely deformed airway. Subsequently, a thorough examination can be done under general anaesthesia via direct laryngoscopy and the laryngeal injury repaired accordingly. The patients are usually put on a feeding tube as to allow adequate healing of laryngeal injuries. Decannulation trials may be successful 2-3 months after injury.

Many patients, however, may recover normal diaphragmatic function in 6-12 months. ${ }^{8}$ Some will recover much earlier with no subsequent complications as seen in our case. A small percentage of patients unfortunately may later present with respiratory or cardiac morbidities. It is therefore advisable to continue seeing these patients longer as not to miss any possible late complications of diaphragmatic trauma and to accommodate the required voice or speech rehabilitation and airway management.

A search of PubMed and OvidSP using the terms "larynx," "laryngeal trauma" and "phrenic nerve" did not yield any report of phrenic nerve injury in association with laryngeal trauma. Given the limited sources, it was a challenge to plan an ideal management of this case. To our knowledge, this is may be the first reported case of phrenic nerve injury in association with blunt laryngeal trauma.

\footnotetext{
REFERENCES

1. Fuhrman GM, Stieg FH $3^{\text {rd }}$, Buerk CA. Blunt laryngeal trauma: Classification and management protocol. J Trauma. $1990 \mathrm{Jan} ; 30(1): 87-92$.

2. Trone TH, Schaefer SD, Carder HM. Blunt and penetrating laryngeal trauma: a 13-year review. Otolaryngol Head Neck Surg. 1980 May-Jun; 88(3):257-61.

3. Iverson LI, Mittal A. DuganDJ, Samson PC. Injuries to the phrenic nerve resulting in diaphragmatic paralysis with special reference to stretch trauma. Am J Surg. 1976 Aug; 132(2):263-269.

4. Nakwan N. A rare case of diaphragmatic paralysis due to isolated phrenic nerve palsy in neonate. SAJCH. 2008 Mar; 2(1): 28-29.

5. Bell D, Siriwardena A. Phrenic nerve injury following blunt trauma. J Accid Emerg Med. 2000 Nov 17(6): 419-420.

6. Snyder RW, Kukora JS, Bothwell WN, Torres GR. Phrenic nerve injury following stretch trauma: case reports. J Trauma. 1994 May; 36(5):734-736.

7. MaziakDE, Maurer JR, Kesten S. Diaphragmatic paralysis: A complication of lung transplantation Ann Thorac Surg. 1996 Jan; 61(1):170-173.

8. Ulku R, Onat S, Balci A, Eren N. Phrenic nerve injury after blunt trauma. Int Surg. 2005 Apr-Jun; 90(2):93-95.

9. McCaul JA, Hislop WS. Transient hemi-diaphragmatic paralysis following neck surgery: report of a case and review of the literature. J.R.Coll.Surg.Edinb. 2001 Jun; 46(3):186-188.

10. Shanmuganathan K, Mirvis SE, White CS, Pomerantz SM. MR imaging evaluation of hemidiaphragms in acute blunt trauma: experience with 16 patients. AJR Am J Roentgenol. 1996 Aug; 167(2):397-402.

11. Nahum E, Ben-Ari J, Schonfeld T, Horev G. Acute diaphragmatic paralysis caused by chest-tube trauma to the phrenic nerve. Pediatr Radiol. 2001 Jun;31(6):444-446.
} 